\title{
A controlled in vitro study of optimal low intensity pulsed ultrasound fields for stimulation of proliferation in murine osteoblasts
}

\author{
Jill Savva \\ School of Engineering \\ University of Glasgow \\ Glasgow, UK \\ j.savva.1@ research.gla.ac.uk
}

\author{
Margaret Lucas \\ School of Engineering \\ University of Glasgow \\ Glasgow, UK \\ margaret.lucas@glasgow.ac.uk
}

\author{
Helen Mulvana \\ Biomedical Engineering \\ University of Strathclyde \\ Glasgow, UK \\ helen.mulvana@strath.ac.uk
}

\begin{abstract}
Clinical, in vivo and in vitro studies have established that Low Intensity Pulsed Ultrasound (LIPUS) stimulates healing of fractured bone, but the mechanisms are not well understood. In vitro studies show cell proliferation, migration and many cellular markers are stimulated by LIPUS at frequencies of $1.0-1.5 \mathrm{MHz}$, even down to $45 \mathrm{kHz}$ [1]. However, most trials did not control or measure the acoustic field, so the dose experienced by the cells across such studies cannot be compared. An in vitro ultrasound exposure method was developed to maintain control of the acoustic field. Murine osteoblasts (MC3T3-E1) were exposed to 20-minute LIPUS fields at the frequencies $1 \mathrm{MHz}$ and $45 \mathrm{kHz}$ and Mechanical Index from 0 (control) to 0.2 . Cell proliferation was assessed by counting viable cells immediately before and twenty hours after LIPUS exposure in the centre of a custom-designed cell culture vessel. Initial results indicate that LIPUS fields increase cell proliferation at $1 \mathrm{MHz}, 0.1 \mathrm{MI}$ and more significantly at $45 \mathrm{kHz}, 0.2 \mathrm{MI}$ compared to controls, but has a detrimental or no effect otherwise. Future work will involve further repeats to acquire larger data sets, along with cell counts in other areas of the cell growth surface to increase data obtained from a single repeat. The study demonstrates the efficacy of the method for quantitative in vitro investigation of LPUS mechanisms and will be used in future work to find optimum LIPUS field characteristics, in terms of frequency, Mechanical Index, and pulse characteristics such as pulse repetition rate and pulse width.
\end{abstract}

Keywords - LIPUS, in vitro, Mechanical Index, MC3T3-E1, osteoblasts, proliferation.

\section{INTRODUCTION}

Low Intensity Pulsed Ultrasound (LIPUS) has been in clinical use for the treatment of non-union fractures (slow-toheal broken bones) for over 20 years, after a number of clinical studies, such as Heckman et al. [2], showed accelerated healing in LIPUS-treated patients compared with controls. Numerous in vitro trials have found that LIPUS stimulates cellular mechanisms involved in bone regrowth, such as cell proliferation and migration of osteoblasts, and the stimulation of mechanotransduction pathways [1]. Most trials to date focussed on LIPUS fields at higher frequencies, typically $1 \mathrm{MHz}$, but low frequency therapeutic fields at $45 \mathrm{kHz}$, produced by devices

This PhD project is funded by the Engineering Physical Sciences

Research Council Doctoral Training Partnership Award 1805089. such as the Duoson (SRA Developments Ltd), have also been shown to have an effect [3]. However, many trials did not quantify and control the acoustic field, resulting in the inability to make accurate comparisons across available data.

In this new study, a protocol for quantitative in vitro investigation of LIPUS was developed. A new cell culture vessel - the 'biocell' - was designed to allow cells to be grown and exposed to known ultrasonic fields. The method was employed to investigate optimum LIPUS fields for cell proliferation in murine osteoblasts. LIPUS fields at $1 \mathrm{MHz}$ and $45 \mathrm{kHz}$, with Mechanical Index from 0 to 0.2 were investigated.

\section{MATERIAL AND METHODS}

\section{A. The biocell - cell culture and ultrasound exposure vessel}

The biocell was developed to allow exposure of a cell monolayer to a known ultrasonic field. The biocell consists of a 3D-printed circular ABS frame forming a 70mm-diameter aperture. $6 \mu \mathrm{m}$-thick, gas-permeable Mylar film (Goodfellow, UK) was stretched across either side to make an optically and acoustically transparent window. Funnels on opposing sides were fitted with self-sealing septa to allow injection of cells and growth media. The funnels were sealed with silicone sealant to ensure a watertight seal. The Mylar growth surface was pretreated with a $10 \mu \mathrm{g} / \mathrm{cm}^{2}$ coating of collagen Type I (from rat tail, Sigma C3867) to improve adhesion.

\section{B. Ultrasonic Field Characterisation}

Two transducers provided ultrasonic fields at $45 \mathrm{kHz}$ and $1 \mathrm{MHz}$. The $45 \mathrm{kHz}$ device was of Langevin design with a cosine-shaped front horn tapering to $2.5 \mathrm{~mm}$ diameter, providing a narrow beam width close to the front face. The $1 \mathrm{MHz}$ device was a $12.7 \mathrm{~mm}$ diameter NDT transducer (V303, Olympus). Both transducers were driven with a signal generator (Agilent 33220A) and power amplifier (E\&I 2200L), through matching transformers.

The pressure field of each transducer was mapped using $2 \mathrm{~mm}(45 \mathrm{kHz})$ and $0.5 \mathrm{~mm}(1 \mathrm{MHz})$ needle hydrophones (Precision Acoustics) in a scanning tank at various distances. 
The Mechanical Index (MI) was derived from the peak-negative pressure in the field by (1).

$$
M I=\frac{p_{-}}{\sqrt{f}}
$$

where $p$. is the peak negative pressure in $\mathrm{MPa}$ and $f$ is the frequency in $\mathrm{MHz}$.

MI is a dimensionless measure used in ultrasound imaging to predict mechanical effects (primarily cavitation) in tissue. The hypothesis here is that it is also an appropriate measure of the extent of excitation of the mechanotransduction pathways involved in LIPUS stimulation.

The acoustic field maps of the $45 \mathrm{kHz}$ and $1 \mathrm{MHz}$ transducers were used to determine positioning of the biocell and dimensions of its acoustically transparent window in order to: (1) allow the main beam (within $10 \mathrm{~dB}$ of the maximum) to pass through unchanged; (2) minimise differences in the 'acoustic treatment zone' while still achieving a maximum of $0.2 \mathrm{MI}$ at the beam centre. The acoustic treatment zone was defined as the $-6 \mathrm{~dB}$ beam width (i.e. the width of the main beam between the points at which the MI dropped to half its maximum value). As a result, the $45 \mathrm{kHz}$ device was characterised at a distance of $6 \mathrm{~mm}$, where the $-6 \mathrm{~dB}$ beam width was $10.5 \mathrm{~mm}$, and the $1 \mathrm{MHz}$ device at $60 \mathrm{~mm}$, where the beam width was $29.4 \mathrm{~mm}$. The beam shapes are shown in Fig. 1.

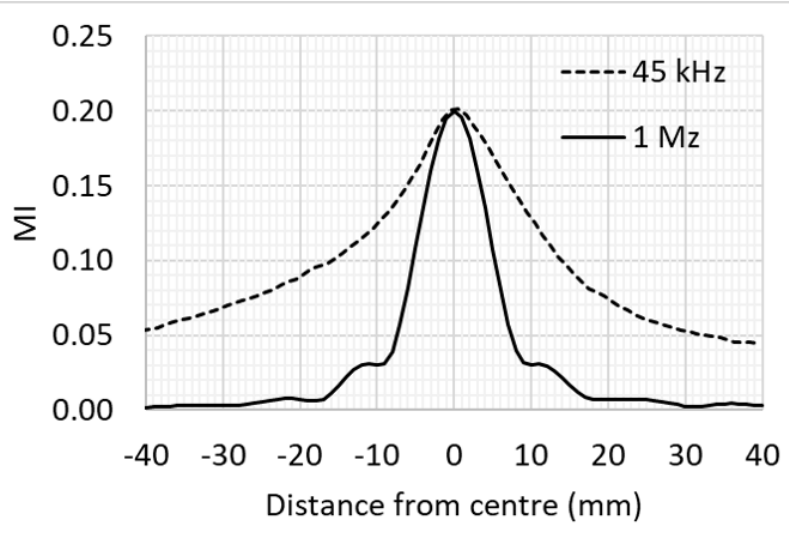

Fig. 1. Beam shapes of $45 \mathrm{kHz}$ and $1 \mathrm{MHz}$ transducers

Thermal effects were assessed by positioning a fibre-optic hydrophone (Precision Acoustics) at the cell growth surface within a biocell and measuring the relative temperature when exposed to each ultrasonic field for 20 minutes. No temperature rise was observed during exposure.

Pressure measurements were conducted through a biocell fitted with one membrane to determine transmission loss. The transmitted pressure was within $\pm 3 \%$ of the free field pressure over the region $\pm 5 \mathrm{~mm}$ around the centre of the biocell window. Thus, it was assumed that the cells were subjected to the same pressures measured in the free field characterisation of the transducers.

\section{Cell Culture}

For each set of ten biocells, one frozen vial of MC3T3-E1 mouse preosteoblast cells (passage 16) were defrosted and grown in tissue culture flasks at a seeding density of 10,000 cells $/ \mathrm{cm}^{2}$, in media consisting of $89 \%$ MEM- $\alpha$ (GIBCO A1049001), 10\% fetal bovine serum (Gibco 10270106) and 1\% Penicillin-Streptomycin (Gibco 15140122). Cells were incubated at $37^{\circ} \mathrm{C}$ and $5 \% \mathrm{CO}_{2}$. Media was renewed every 2-3 days and cells were detached with Trypsin-EDTA $0.25 \%$ (Sigma T4049). The biocells were seeded at passage 18 with seeding density 25,000 cells $/ \mathrm{cm}^{2}$.

\section{Cell Proliferation}

Cell proliferation was quantified by comparing viable cell counts immediately before and 20 hours after ultrasound exposure. The media was removed from each biocell and replaced with media containing fluorescent dyes from the Readyprobes ${ }^{\mathrm{TM}}$ cell viability kit (Invitrogen ${ }^{\mathrm{TM}} \mathrm{R} 37610$ ). At each point of interest, two fluorescent images were captured: (1) all cells live or dead (Blue), and (2) dead cells (Red). Five images were captured using the $\times 10$ lens at the centre and in four immediately adjacent areas, illustrated in Fig 2. The position of the centre point was determined by observation of the position of the bright field light source on the cell growth surface and aligning this centrally with reference to the funnel positions.

The image dimensions captured were $486 \mu \mathrm{m}$ width by $362 \mu \mathrm{m}$ height, an area of $0.00176 \mathrm{~cm}^{2}$ (containing approximately 44 cells at point of seeding). The total extent of captured images was within $\pm 0.55 \mathrm{~mm}$ and $\pm 0.75 \mathrm{~mm}$ of the centre. Taking positioning errors into account, the total error was estimated to be a maximum of $\pm 2 \mathrm{~mm}$, corresponding to an error in MI exposure of $\pm 10 \%$.

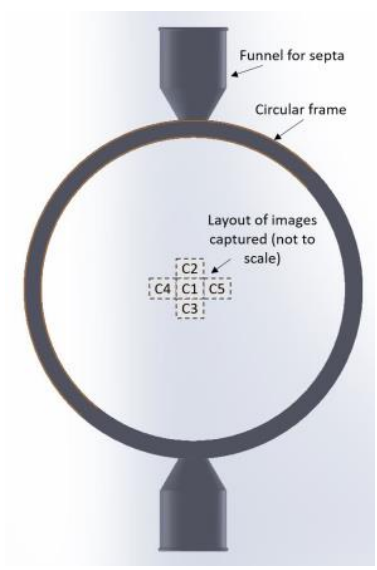

Fig. 2. Schematic of image capture positions on the biocell for viable cell counting (not to scale)

Cell counts were automated in ImageJ [4] and the dead cell counts subtracted from live/dead cell counts to give the number of viable cells. The average viable cell count from all captured images before and after LIPUS exposure were then compared. Total cell counts in each biocell were also estimated using a hemocytometer.

\section{E. In vitro ultrasound exposure}

A single ultrasound exposure test set consisted of ten biocells. Each biocell was seeded, filled with media and incubated overnight. Immediately prior to ultrasound exposure the media was removed, and viable cells counted with the ReadyProbes kit. After removal of the dye/media solution the biocell was washed with DPBS and the media refreshed. 
Ultrasound exposure was conducted in a tank of deionised, degassed, sterilised water at a temperature of $33 \pm 1{ }^{\circ} \mathrm{C}$. An acoustic absorber placed at one end of the tank eliminated reflections. Each biocell was positioned at the required distance from the test transducer, aligning against marked centre-lines and exposed to 20 minutes of LIPUS at either $1 \mathrm{MHz}$ or $45 \mathrm{kHz}$ with a Mechanical Index of 0 (control), $0.05,0.1,0.15$ or 0.20 . The pulse characteristics were the same in all cases, with pulse width $200 \mu \mathrm{s}$ and pulse repetition rate $1 \mathrm{kHz}$. Controls underwent the same protocol but with no emitted drive signal. Post-exposure, the biocells were removed from the tank and returned to the incubator. Twenty hours post-exposure, cell viability counts were repeated and a final hemocytometer cell count conducted. The process was repeated two times.

\section{RESULTS}

Fig. 3 shows the average viable cell counts immediately before exposure and 20 hours post-exposure for both frequencies of interest.

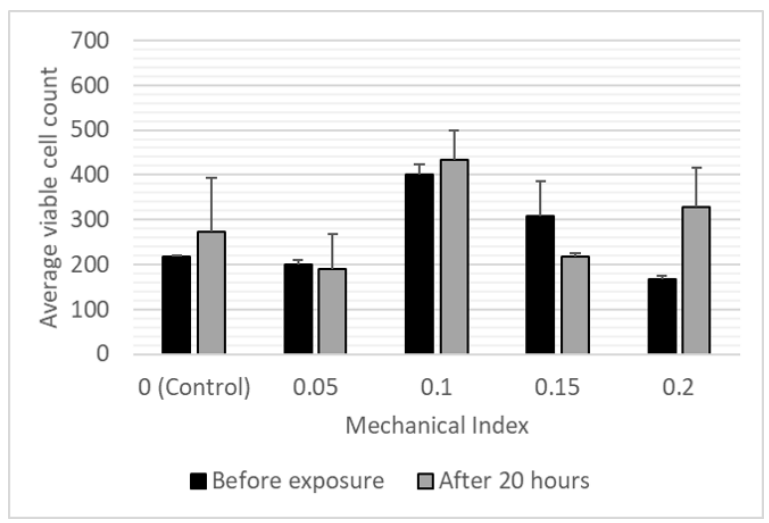

(a)

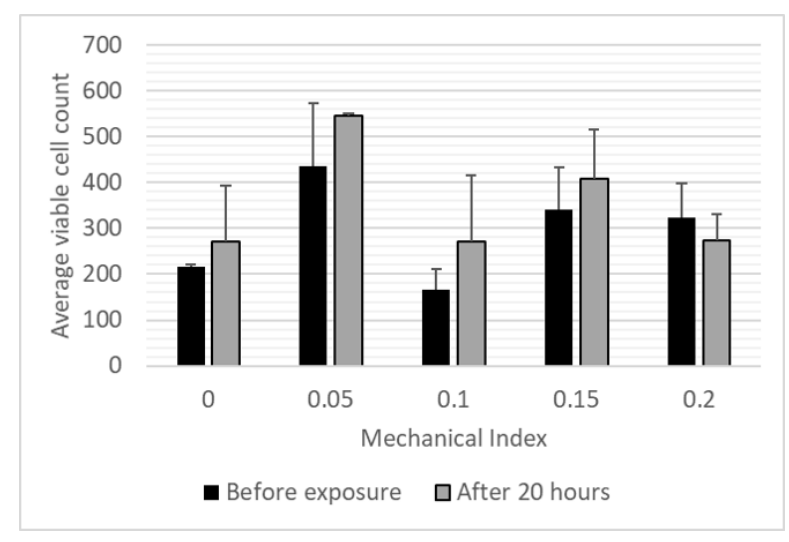

(b)

Fig. 3. Average viable cell counts in microscope images immediately before exposure and 20 hours after exposure at (a) $45 \mathrm{kHz}$ and (b) $1 \mathrm{MHz}$, with error bars indicating standard deviation $(\mathrm{n}=2)$.

The percentage differences between 'before' and 'after' viable cell counts were calculated to indicate the rate of cell proliferation. The results are presented in Fig. 4.

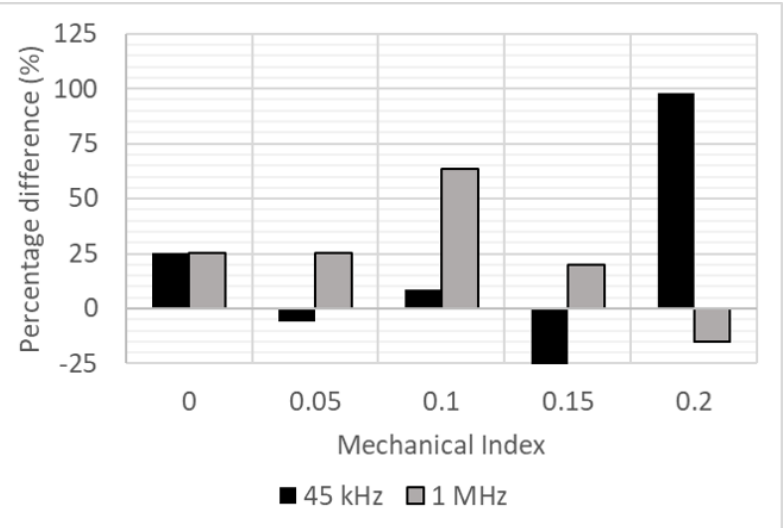

Fig. 4. Percentage difference in average viable cell counts in microscope images immediately before and 20 hours after exposure to LIPUS fields

\section{DISCUSSION AND CONCLUSIONS}

The results suggest that $1 \mathrm{MHz}$ LIPUS significantly increases proliferation compared with controls at $0.1 \mathrm{MI}(63 \%$ increase compared with $25 \%$ in controls). Percentage differences at all other MI settings were equal to or less than controls. An MI of 0.2 had a detrimental effect on cell proliferation, with a reduction of $15 \%$ in viable cells. This could be a result of cell death, or of cells losing adhesion and being washed off during DPBS washes and media changes.

While the $1 \mathrm{MHz}$ results suggest a smooth transition between enhancement and detriment of cell proliferation, the $45 \mathrm{kHz}$ results are not so straightforward. At MI less than 0.2, viable cell numbers were consistently reduced: the worst-case decrease was $29 \%$ at 0.15 MI. However, a significant increase of $98 \%$ was observed in viable cells at $0.2 \mathrm{MI}$. This suggests that low frequency LIPUS requires higher MIs to produce a stimulatory effect, but this effect is stronger than observed at $1 \mathrm{MHz}$.

The differences between low and high frequency LIPUS may be due to the envelope of the low frequency pulse. The higher frequency pulse had a shorter rise time, resulting in an effective switch on / switch off of radiation force. This sudden switching may cause damage to the cells or affect their adhesion to the membrane. In contrast the low frequency device had a smoother transition to the maximum MI. The reason for reduced cell proliferation at lower MIs in the low frequency results is unknown and requires further investigation.

The method shows promise in enabling controlled in vitro study of LIPUS fields. With careful design of the aperture diameter, the new culture vessel allows an ultrasonic field to be applied to a cell monolayer without significant change to the acoustic field measured in free field conditions. The frame is 3D printed and therefore cheap to produce, and it can be customised to accommodate varying beam widths. After careful cleaning the frames can be re-used. However, constructing the biocells is a lengthy process. Using a single culture vessel also makes the large number of repeats required for in vitro testing time consuming. The small sample set $(\mathrm{n}=2)$ achieved so far resulted in standard deviations up to $54 \%$ in average viable cell counts. 
Consequently, the reported results only provide an indication of effects of LIPUS on cell proliferation. Further repeats are required to increase the sample set and reduce uncertainty.

Fluorescence microscopy was employed to count viable cells in the centre of the acoustic beam, where cells experienced the maximum MI. The use of fluorescence microscopy has its drawbacks as it involves exposure of cells to harmful UV light. The intensity of light and duration of exposure were kept to a minimum in this study to minimise potential adverse effects on cells.

Cell proliferation alone is an incomplete indicator of the healing capacity of a LIPUS field. To assess this, upregulation of cellular markers such as Prostaglandin E2 (PGE2), a substance produced by one of the mechanotransduction pathways linked to LIPUS [1], will be compared to global acoustic parameters such as the spatially averaged MI, to determine potential healing effects. In the future we intend to use PGE2 concentration to investigate the impact of LIPUS pulse width and repetition rate on cells. Future work will also study cell proliferation in other areas of the biocell, which are exposed to lower MIs within the acoustic beam.

In conclusion, the study has demonstrated that the in vitro ultrasound exposure method presented allows controlled investigation of the effects of known LIPUS fields on cell monolayers. Early results suggest that LIPUS fields at low frequency and high frequency can stimulate cell proliferation, with optimum results observed at $0.2 \mathrm{MI}(45 \mathrm{kHz})$ and $0.1 \mathrm{MI}$ $(1 \mathrm{MHz})$. Further repeats are required to assess the significance of the initial findings.

\section{ACKNOWLEDGMENTS}

The authors wish to thank Dr Sarah Brueningk and Professor Gail ter Haar of the Institute of Cancer Research for their advice and expertise on in vitro testing.

\section{REFERENCES}

[1] F. Padilla, R. Puts, L. Vico, K. Raum, "Stimulation of bone repair with ultrasound: A review of the possible mechanic effects." Ultrasonics, 2014 54(5): p. 1125-1145.

[2] J. D. Heckman, J. P. Ryaby, J. McCabe, J. J. Frey, and R. F. Kilcoyne, "Acceleration of Tibial Fracture-Healing by Non-Invasive, Low-Intensity Pulsed Ultrasound," The Journal of Bone and Joint Surgery, vol. 76-A, pp. 26 - 34, January 1994.

[3] J. Man, P. R. Cooper, B. A. Scheven, R. M. Shelton, and G. Landini, "Low Intensity Ultrasound stimulates osteoblast migration at different frequencies," The Japanese Society for Bone and Mineral Research and Springer, vol. 2012, pp. 602-607, January 2012.

[4] C. A. Schneider, W. S. Rasband, and K. W. Eliceiri, "NIH Image to ImageJ: 25 years of image analysis," Nature methods, vol. 9, p. 671, 2012. 